\title{
Hematuria in paediatrics
}

\section{Maya Mouzannar}

Bahman University Hospital, Lebanon

*Corresponding author: Maya Mouzannar, MD Pediatrician, Bahman University Hospital, Lebanon, Tel: 96170963605; E-mail: M.Mzanar@googlemail.com Received date: May 21, 2018; Accepted date: May 26, 2018; Published date: May 31, 2018

Copyright: (c) 2018 Mouzannar M, This is an open-access article distributed under the terms of the Creative Commons Attribution License, which permits unrestricted use, distribution, and reproduction in any medium, provided the original author and source are credited.

\section{Case Review}

\section{Case 1}

A 10 year-old boy presented to the pediatric clinic with gross hematuria, tea-colored urine dating from 4 days before consulting, mild pelvic abdominal pain, decreased appetite and history of 2 days of fever prior, no nausea, no vomiting.

Upon reviewing the past medical history over the previous weeks: patient noted that 15 days prior to consulting, he complained of sore throat with enlarged tonsils, no antibiotic was taken. On physical exam, patient has a blood pressure of $115 / 79$, bilateral flank abdominal tenderness, no edema.

Urine analysis done showed leucocyturia, hematuria, no casts, trace of protein. Abdominal, renal and pelvic ultrasound examinations are found normal. Blood test showed a white count of $12100 / \mathrm{uL}$, hemoglobin of 13, $4 \mathrm{~g} / \mathrm{dl}$, creatinine of $0,53 \mathrm{mg} / \mathrm{dl}$, BUN 12, $68 \mathrm{mg} / \mathrm{dl}$, sodium of $139 \mathrm{mmol} / \mathrm{L}$, potassium of $5.38 \mathrm{mmol} / \mathrm{L}$, carbon dioxide of $19.53 \mathrm{mmol} / \mathrm{L}$, urine culture showed no growth. Other tests prescribed not done for financial reasons. Patient received 10 days of cefixime and noted improvement upon day 3. Patient was lost to follow-up.

\section{Case 2}

A 10 year-old girl presented to the pediatric clinic with abdominal pain and sore throat. On exam, she has a blood pressure of 111/80, left flank and right flank tenderness, no lower limbs edema. Strep test done was negative (repeated twice). Patient has family history of kidney stones. Urine analysis done showed 20-25 RBC, no proteinuria, no leucocyturia, and urine culture showed no growth. Urine analysis was repeated at 4 weeks interval then 2 months interval, showed persistant microscopic hematuria 18-20 RBC, no casts, no proteinuria. Ultrasound exam of the abdomen and pelvis was unremarkable. Blood test showed hemoglobin of $12.6 \mathrm{~g} / \mathrm{dl}$, WBC $7130 / \mathrm{uL}$, creatinine 0.33 $\mathrm{mg} / \mathrm{dl}$, BUN $10 \mathrm{mg} / \mathrm{dl}$.

Hematuria is a common finding in the pediatric population. It can present as the reason for consultation or an incidental finding on routine urinalysis done in the evaluation of abdominal pain or urinary tract symptoms [1]
The objective of this article is to help the general practitioner define hematuria, plan the approach to the evaluation and management and identify when a referral to a pediatric nephrologist is required.

Hematuria can be defined as "the presence of 5 or more red blood cells (RBCs) per high-power field in 3 of 3 consecutive centrifuged specimens obtained at least 1 week apart. Hematuria can be either gross (bloody, smoky, or tea-colored urine) or microscopic. It may also be either symptomatic or asymptomatic, either transient or persistent, and either isolated or associated with proteinuria and other urinary abnormalities" [2].

A prompt evaluation is required in the case of gross hematuria. A urinalysis is ordered. If the urine is red and there are no red blood cells (RBCs) on microscopy, this suggests a possible food, medication or chemical ingestion causing the coloration or the presence of free haemoglobin or myoglobin. Acute intravascular hemolysis, disseminated intravascular coagulation can cause hemoglobinuria. Rhabdomyolysis, burn myositis or asphyxia may cause myoglobinuria.

In infants, if an orange-red color is found in diapers, it is a possible precipitation of urate crystals [3].

Examining urinary RBCs morphology helps you navigate the possible cause of hematuria and the orientation in ordering the required diagnostic tests.

Dysmorphic RBCs are typically detected in glomerular causes. However their absence does not rule out glomerular origin.

It is essential to rule out these possible causes:

Immunological glomerular injury including the following: post streptococcal acute glomerulonephritis, Alport syndrome.

Infectious glomerulonephritis

Hemolytic uremic syndrome [3].

Urinary tract infection is a common identifiable cause of hematuria. Hematuria without casts is noted in sickle cell trait or disease, after strenuous exercise and after renal trauma.

For the evaluation of a child with hematuria, please refer to Table 1 and Figures 1 and 2. A renal biopsy is warranted in the case of hematuria and significant proteinuria [3].

\footnotetext{
Evaluation of a child with hematuria

1. Complete history and physical examination (Particularly blood pressure, optic discs, skin, abdomen genitalia).

2. Confirmation of true hematuria by urine microscopic examination.

3. Urine culture

4. Urine Calcium, Protein, Creatinine
} 


\begin{tabular}{|ll|}
\hline 5. & Complete blood count including platelets, serum electrolytes, BUN/ serum Creatinine (calculate Creatinine clearance), calcium total protein and albumin. \\
\hline 6. & Streptozyme, C3, C4, ANA \\
\hline 7. & Renal Ultrasonography \\
\hline 8. & Renal Biopsy in selected cases \\
\hline Abbreviations: Antinuclear Antibody (ANA); Blood Urea Nitrogen (BUN); Complement Component 3 (C3); Complement Component 4 (C4) \\
\hline
\end{tabular}

Table1: Evaluation of a child with hematuria

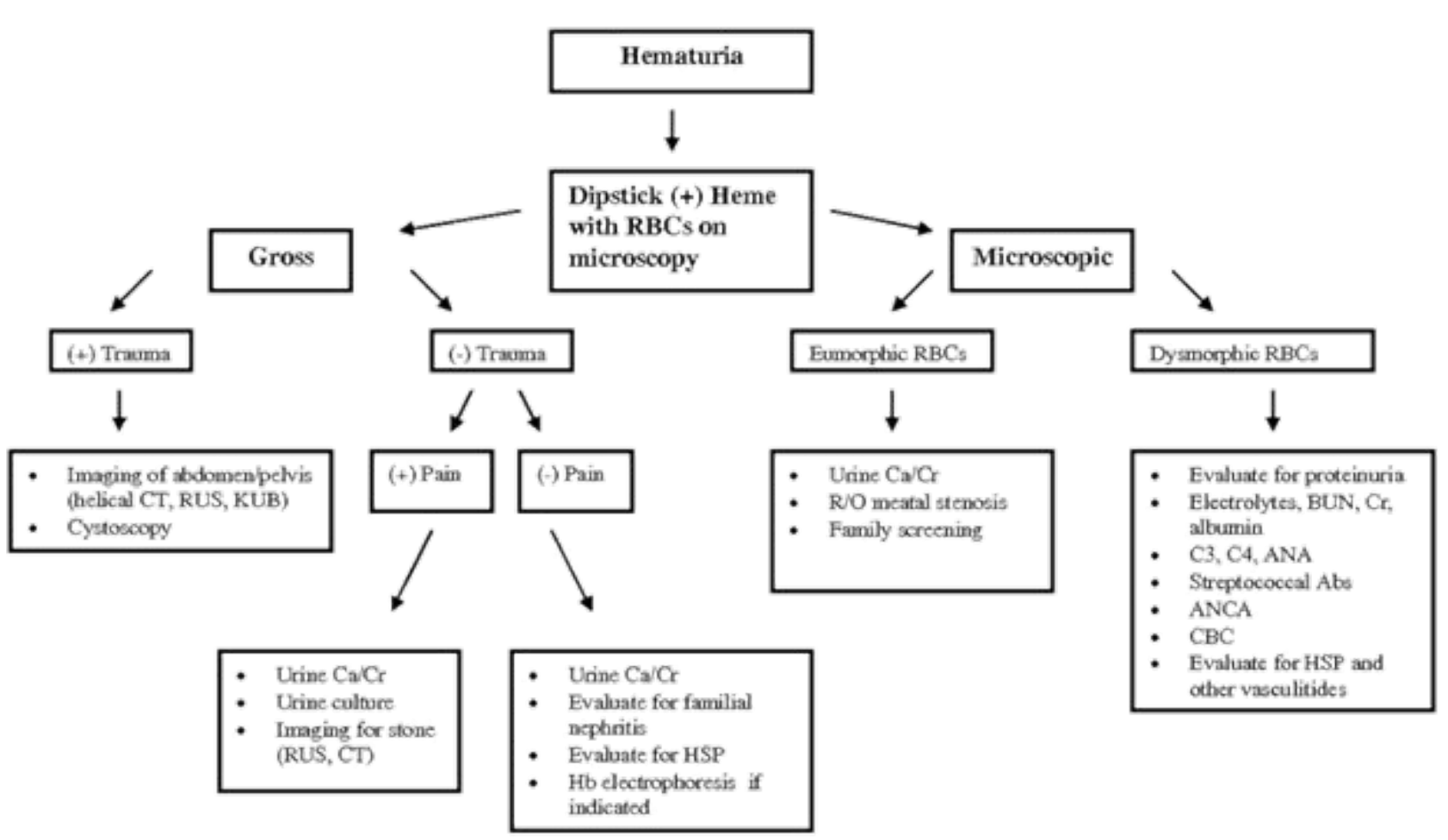

Figure 1: Algorithm of evaluation of hematuria.

A retrospective study published by Feld and al. in 1998, of 325 children referred for hematuria, concluded to the following: a renal ultrasound, voiding cystourethrogram, cystoscopy, and renal biopsy are not indicated in the work-up of microscopic hematuria, and microhematuria in the otherwise healthy child is a minimal health threat, rarely indicative of serious illness [4]. The authors published an algorithm for the follow up of patients found to have microscopic hematuria (Figure 2). A family history of renal stones and a urine calcium to creatinine ratio below or above 0.2 were the key determinants for differentiating between familial microscopic hematuria (a ratio below 0.2 ) and possible hypercalciuria.

Treatment varies from dietary restrictions, sodium restrictions, diuretics, antihypertensive agents, corticosteroids, immunosuppressive therapy and anti-inflammatory agents.

\section{Discussion}

The first clinical case is that of gross hematuria with eumorphic RBCs in urine, leucocyturia trace of proteinuria, abdominal pain and history of tonsillitis. A urinary tract infection and kidney stones were ruled out. Patient improved on antibiotic therapy. Was it a urinary tract infection with a negative culture or post streptococcal acute glomerulonephritis?

The second clinical case is that of microscopic hematuria with family history of kidney stones, abdominal pain with no trauma. A urinary tract infection and kidney stones were ruled out. A urine calcium to creatinine ratio is required to help differentiate between familial microscopic hematuria and possible hypercalciuria. 


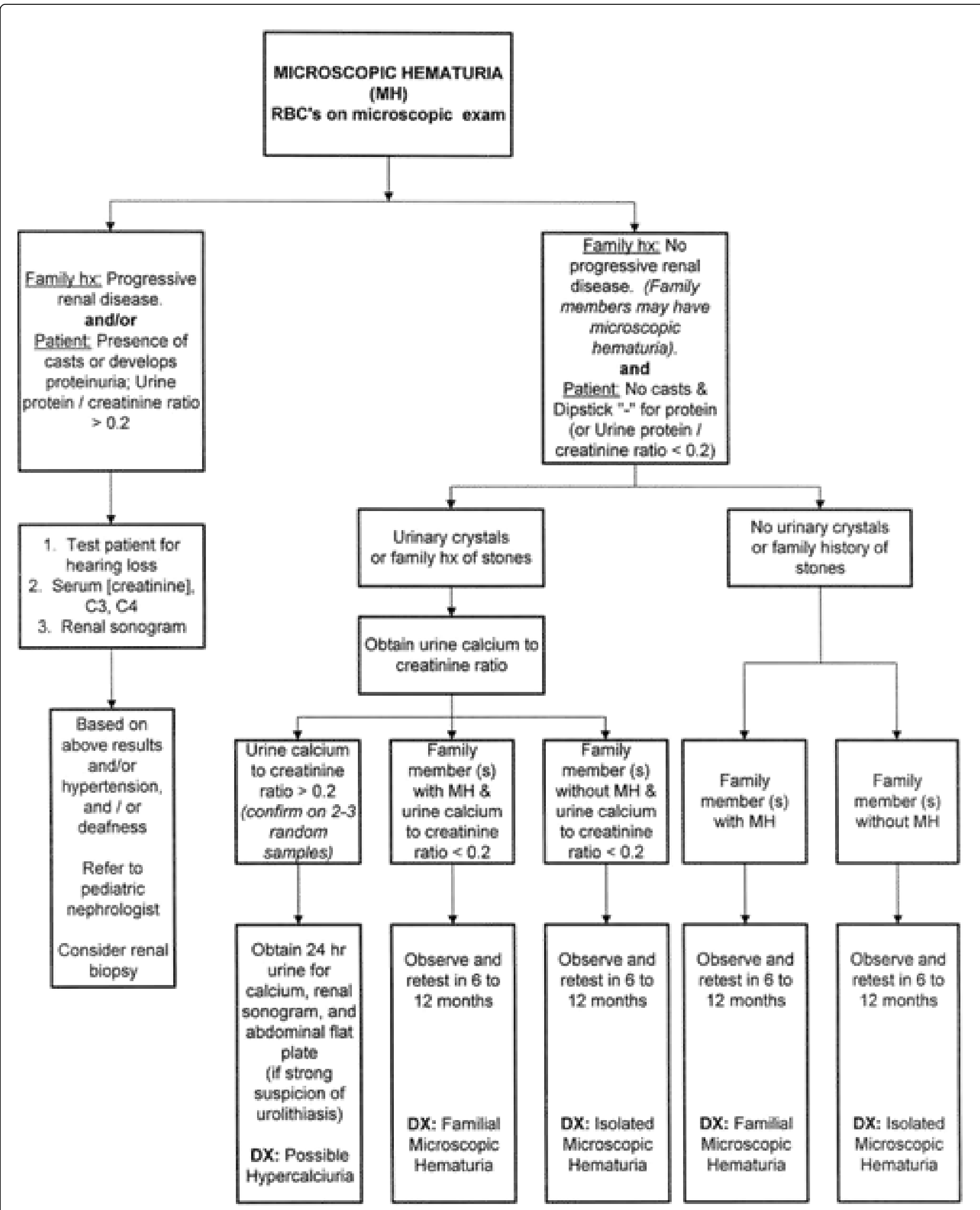

Figure 2: Algorithm of management of microscopic hematuria. 
Page 4 of 4

It is not evident to manage pediatric hematuria but with the right tools especially algorithms, any general practitioner can be well oriented in the diagnosis and management.

\section{References}

1. Massengill SF (2008) Hematuria. Pediatr Rev 29: 10.

\section{Gulati S (2017) Hematuria. Medscape.}

3. Marcdante K. Kliegman R, Jenson H., Behrman R (2011) Chapter 163 Glomerulonephritis and Hematuria. Nelson Essentials of Pediatrics 6th Edition. Saunders Elsevier.

4. Feld LG, Meyers KE, Kaplan BS, Stapleton FB (1998) Limited Evaluation of Microscopic Hematuria in Pediatrics. Pediatrics 102: E42. 